\title{
Thinking the Impossible
}

\author{
Graham Priest
}

March 25, 2016

Departments of Philosophy, Graduate Center, City University of New York, and the University of Melbourne

Possible? Is anything impossible? Read the newspapers. Arthur Wellesley (Duke of Wellington). ${ }^{1}$

\section{Introduction: the History of Modality}

Possibility has been a familiar character in Western philosophy since the inception of the discipline. Systematic ways of thinking about it are to be found in both of the first two great periods of logic: Ancient and Medieval. Witness Aristotle's modal syllogistic, ${ }^{2}$ and and its medieval developments, such as the doctrine of ampliation, and the notions of sensu composito and sensu diviso. ${ }^{3}$

The articulation of the notion has taken a very distinctive turn in the third great (and contemporary) period. Possible-world semantics has come to take centre stage. And the applicability of these has spread the tentacles of modality into areas where connections had not before been made, such as meaning, belief, conditionals, and intentionality. ${ }^{4}$ Of late, we have seen an extension of this logical technology into the area of impossible worlds,

\footnotetext{
${ }^{1}$ Cohen and Cohen (1992), p. 450.

${ }^{2}$ See Smith (2011).

${ }^{3}$ See Knuuttila (2014).

${ }^{4}$ See Garson (2014).
} 
stretching the tentacles further, and-arguably - untangling some knots in the earlier tentacles. ${ }^{5}$

The present paper takes a close look at the structure of impossible worlds, and of one in particular of the tentacles: the mental state of conception/imagination.

\section{Possible Worlds}

\subsection{Their Structure}

Possible-world semantics are familiar to contemporary logicians and philosophers, and need little explanation; ${ }^{6}$ but let me set things up in a slightly unusual way, for reasons that will become clear later. Take a propositional language with the connectives: $\wedge, \vee, \neg, \diamond, \square .^{7}$ ( $\supset$ can be defined in the usual way.) An interpretation for the language has four components: $\langle X, R, @, \nu\rangle$. $X$ is a set of (possible) worlds. (It would be more normal to write this as $W$; but I will hold this letter in reserve till later.) $R$ is a binary relation on $X$ : relative possibility. @ $\in X$ is the actual world. And $\nu$ assigns every propositional parameter a pair of subsets of $X, \nu^{+}(p)$ and $\nu^{-}(p)$, subject to the constraints of exclusivity and exhaustivity:

Exc: $\nu^{+}(p) \cap \nu^{-}(p)=\emptyset$

Exh: $\nu^{+}(p) \cup \nu^{-}(p)=X$

Intuitively, $\nu^{+}(p)$ is the set of worlds where $p$ is true; $\nu^{-}(p)$ is the set of worlds where $p$ is false.

We now define what it is for a formula to be true $\left(\Vdash^{+}\right)$and false $\left(\Vdash^{-}\right)$at a world $w \in X$ :

- $w \Vdash^{+} p$ iff $w \in \nu^{+}(p)$

- $w \Vdash^{-} p$ iff $w \in \nu^{-}(p)$

- $w \Vdash^{+} \neg A$ iff $w \Vdash^{-} A$

- $w \Vdash^{-} \neg A$ iff $w \Vdash^{+} A$

\footnotetext{
${ }^{5}$ See Berto (2014).

${ }^{6}$ See Priest (2008), chs. 2, 3.

${ }^{7}$ What follows applies equally to a first-order languages, but their specificities are not relevant to the following considerations.
} 
- $w \Vdash^{+} A \wedge B$ iff $w \Vdash^{+} A$ and $w \Vdash^{+} B$

- $w \Vdash^{-} A \wedge B$ iff $w \Vdash^{-} A$ or $w \Vdash^{-} B$

- $w \Vdash^{+} A \vee B$ iff $w \Vdash^{+} A$ or $w \Vdash^{+} B$

- $w \Vdash^{-} A \vee B$ iff $w \Vdash^{-} A$ and $w \Vdash^{-} B$

- $w \Vdash^{+} \diamond A$ iff for some $w^{\prime}$ such that $w R w^{\prime}, w \Vdash^{+} A$

- $w \Vdash^{-} \diamond A$ iff for all $w^{\prime}$ such that $w R w^{\prime}, w \Vdash^{-} A$

- $w \Vdash^{+} \square A$ iff for all $w^{\prime}$ such that $w R w^{\prime}, w \Vdash^{+} A$

- $w \Vdash^{-} \square A$ iff for some $w^{\prime}$ such that $w R w^{\prime}, w \Vdash^{-} A$

Validity $(\models)$ is defined as preservation of truth at @ in every interpretation. ${ }^{8}$

A simple induction shows that for every formula, $A$, and world, $w, w \Vdash^{+} A$ or $w \Vdash^{-} A$, but not both. Hence, given that no constraints are placed on $R$, the logic delivered is simply the modal logic $K$.

It should be noted that an interpretation is simply a piece of mathematical machinery. In particular, $X$ is any old set of objects. These are not to be confused with possible worlds themselves. We may naturally suppose, however, that there is one interpretation of the language which is in accord with the real. (In this, $X$ is the set of real possible worlds, @ is the real actual world, $R$ is the real relation of relative possibility, and $\nu^{+}(p) / \nu^{-}(p)$ are the sets of worlds in which $p$ - understood as some meaningful sentence - is really true/false.) That is why we can reason using modal logic about reality (not just actuality: actuality is just one world of the plurality of worlds). Why do we require a plurality of interpretations to define validity? For the same reason that we do in the case of propositional non-modal logic. We want our inference relation to be applicable whatever reality is, in fact, like.

Of course, none of this tells us what kind of thing possible worlds are. Physical objects or abstract objects, existent objects or non-existent objects? There are many well-known accounts of this matter; ${ }^{9}$ and which is right need not concern us in this essay. Of more concern here is possibility itself.

\footnotetext{
${ }^{8}$ In some standard presentations, there is no designated world, @, and validity is defined as truth preservation over all possible worlds. As long as no special constraints are put on @, this is, of course, equivalent. However, it will be useful in what follows to have @ at our disposal.

${ }^{9}$ See Menzel (2013). My own account can be found in Priest (2005), 7.3.
} 


\subsection{A Plurality of Possibilities}

Possibility comes in many flavours. To name but a few: physical $(\varphi)$, epistemic $(\varepsilon)$, denontic $(\delta)$. Let us write $K$ for the set of different kinds of possibility. For every member of $K$ there will be a corresponding notion of necessity. If $\kappa \in K$, let us write the corresponding modal operators as $\langle\kappa\rangle$, and $[\kappa]$. In an interpretation, each $\kappa \in K$ will also have its own accessibility relation, $R_{\kappa}$. Thus we will have for each $\kappa$ :

- $w \Vdash^{+}\langle\kappa\rangle A$ iff for some $w^{\prime}$ such that $w R_{\kappa} w^{\prime}, w \Vdash^{+} A$

- $w \Vdash^{-}\langle\kappa\rangle A$ iff for all $w^{\prime}$ such that $w R_{\kappa} w^{\prime}, w \Vdash^{-} A$

- $w \Vdash^{+}[\kappa] A$ iff for all $w^{\prime}$ such that $w R_{\kappa} w^{\prime}, w \Vdash^{+} A$

- $w \Vdash^{-}[\kappa] A$ iff for some $w^{\prime}$ such that $w R_{\kappa} w^{\prime}, w \Vdash^{-} A$

What we have now done is to move from a mono-modal logic to a multimodal logic, whose language contains a multiplicity of possibility/necessity operators, and whose semantics contains a corresponding multiplicity of accessibility relations. ${ }^{10}$

The accessibility relations will come with appropriate constraints. Thus, one would expect that for every world, $w, w R_{\varepsilon} w$, so that $[\varepsilon] A \models A$ (what is known is true). Sometimes, the accessibility relations will be nested, in the sense that possibility in one sense implies possibility in another. (We will have an example of this in a moment.) Sometimes there is no nesting. For example, epistemic and physical possibility properly overlap. Thus, there is a physical limit to how fast a marathon can be run. Suppose, for the sake of argument, that this is one hour. Then it is physically possible to run a marathon in 61 minutes, and physically impossible to run a marathon in 59 minutes. But both of these are (currently) epistemic possibilities. Conversely, it is both physically possible and epistemically possible for something to be made of antimatter. In the 13th Century, it was still a physical possibility, but it was not an epistemic possibility: people then had no conception of antimatter, or, therefore, of its possibilities.

For the most part, we will not be concerned with the constraints on the accessibility relations - with one major exception. Given possible-world semantics, there is a most general notion of possibility. To be possible in

\footnotetext{
${ }^{10}$ See Carnielli and Pizzi (2008).
} 
this sense is simply to hold at some world. How to describe this kind of modality, one might argue about. I shall simply call it logical, $\lambda$. (Though it is worth noting that logical necessity in this sense will include things that are not formally logically necessary, including analytic truths such as 'all red things are coloured' and mathematical truths such as 'there is an infinitude of prime numbers'.) Anything that is possible in any more restricted sense is possible in this sense; and $R_{\lambda}$ is simply the universal relation: every possible world accesses every other. Hence, for any $\kappa \in K$, we have:

- $\langle\kappa\rangle A \models\langle\lambda\rangle A$

- $[\lambda] A \models[\kappa] A$

and the modal logic of $\lambda$ is $S 5$.

Here we see the beginning of a problem. Some things that are epistemically possible would seem to be logically impossible. Thus, before Wiles' proof of the truth of Fermant's last theorem, its negation was epistemically possible, though logically impossible.

\section{Impossible Worlds}

\subsection{The Primary Directive}

The rediscovery of modal logic in the 20th century was in the work of C. I. Lewis between the two World Wars. Possible-world semantics came to prominence in the 1960s and 70s. At first, under the influence of Quine's attack on things modal, possible worlds and their machinations were considered creatures of darkness. But the clarity of the mathematics involved, and their usefulness in an analysis of many things other than modality - such as conditionals, meaning, knowledge and belief - meant that they soon became part of the intellectual landscape. The philosophical debate around worlds changed from whether one can make sense of them to how best to make sense of them, given the slew of theories about their nature.

Impossible worlds rose to prominence some 20 years later. Under a very different ideology (that of the unintelligibility of inconsistency), they, too, were often taken to be creatures of darkness. Current debates may still concern whether one can make sense of them. However, their mathematics is clear, and their applicability to many philosophical areas-including some 
of those in which possible worlds were clearly problematic - have, I think, ensured, that they will soon be as much part of the landscape as possible worlds. $^{11}$

Of course, for certain notions of possibility, impossible worlds can be accommodated in possible-world semantics. Thus, physically impossible worlds, where, say, a particle accelerates through the speed of light, are logically possible; and so can be accommodated in a model which allows for all logical possibilities.

The main problem is with logical impossibilities themselves. On standard possible-world semantics there are no worlds which realise these. But if there are worlds which do so, there must be worlds where logical impossibilities hold; and dually, worlds where logical truths fail. There appears to be no reason to distinguish between different kinds of logical truths and falsehoods in this regard. Hence we have the first leading principle of impossible-world semantics: any logical truth must fail at some worlds, and any logical falsity must hold at some worlds. Of course, this was already the case for logically contingent things. Hence we arrive at what we may call the Primary Directive:

- Everything holds at some worlds, and everything fails at some worlds.

What, then, is the technology of logically impossible worlds? We may simply broaden the class of worlds by dropping Exc and Exh.

It is almost trivial to check that if there are no constraints on accessaibility relations at impossible worlds the Primary Directive is then satisfied. Take any formula, $A$, and consider a world, $w$, such that for all $\kappa \in K, w$ accesses itself and only itself under $R_{\kappa}$, and for every parameter, $p$, in $A, w \in \nu^{+}(p) \cap$ $\nu^{-}(p)$. A simple induction shows that every formula whose parameters are amongst the $p \mathrm{~s}$ - and so $A$-is both true and false at $w$. Dually, replace the condition $w \in \nu^{+}(p) \cap \nu^{-}(p)$ with $w \notin \nu^{+}(p) \cup \nu^{-}(p)$, and a similar induction shows that $A$ is neither true nor false at $w .^{12}$

\footnotetext{
${ }^{11}$ On these issues, see Priest (1997a) and Berto (2013).

${ }^{12}$ I note that if we make all the propositional parmeters true and false at $w$, then every formula is true there; and if we make all the propositional parameters neither true nor false at $w$, all formulas are neither true nor false there. Hence, the primary directive can, in fact, be satisfied with just these two worlds. However, the two, on their own, hardly do justice to the diversity of impossible situations.
} 


\subsection{Possibile Worlds Revisited}

So far so good. Let $P \subseteq X$ be the logically possible worlds. We should clearly require that $@ \in P$. (What is actual is logically possible.) And we should require that the worlds in $P$ access each other, and nothing else, under $R_{\lambda}$.

But what is $P$ ? One reason the answer is important is that it determines the validity relation, since this was defined in terms of truth preservation at @, and @ is in $P$. Those who think that classical logic gets the validity relation right will, of course, suggest that $P$ is a proper subset of $X$, namely, the worlds where Exh and Exc hold. These are the worlds closed under classical S5 for the modality $\lambda$, except the trivial world (where everything is true). ${ }^{13}$

What if one does not think that classical logic is correct? If one takes $F D E$ (First Degree Entailment) to be the correct logic, then we could, at the other extreme, as it were, just take $P$ to be $X$ itself (so there are no impossible worlds - at least of this kind; stay tuned). There are intermediate possibilities. If one takes the correct logic to be $L P$, we will just reinstate the condition Exh. The possible worlds are then those that are closed under $L P$ $S 5$ consequence (including, NB, the trivial world). Alternatively, if one takes the correct logic to be $K_{3}$, we will reinstate the condition Exc. The possible worlds are then those closed under $K_{3}-S 5$, except the trivial world. ${ }^{14}$ I note that the Primary Directive may no longer be satisfied for modal formulas involving $\lambda$. Thus, in $L P \square(p \vee \neg p)$ will hold at all worlds; and in $K_{3}$ $\diamond(p \wedge \neg p)$ will fail at all worlds. This will be rectified with the Secondary Directive, as we shall see in a moment.

It is sometimes touted as a virtue of possible-world semantics that they provide a reductive account of (logical) possibility. To be possible is simply to hold in some world. Exactly the same is true if $P$ is $X$; but of course, it is not true if $P$ is a proper subset of $X$. Reduction has alway struck me as a dubious virtue, however. Why should one expect such a reduction? We obviously don't have it for all the other kinds of possibility; why just this

\footnotetext{
${ }^{13}$ Any such world is obviously closed under the consequence relation. Conversely, if $w$ does not satisfy $\mathbf{E x h}$ and $\mathbf{E x c}$, it is clearly not closed under the relation. If it accesses a world, $w^{\prime}$, that is not so closed, then for some, $p, p$ is either both true and false at $w^{\prime}$ or neither true nor false there. In the first case, $\diamond(p \wedge \neg p)$ is true at $w$; but in S5, $\diamond(p \wedge \neg p) \models A$, so the world is either not closed under the consequence relation, or is trivial. In the second case, it is not true that $\square(p \vee \neg p)$ at $w$, so the world is not closed under Necessitation.

${ }^{14}$ For these non-classical modal logics, see Priest (2008), ch. 11a.
} 
one? Or better, we have to give a non-reductive account for the other notions of possibility, so we ought to be able to do it for this one too. We have just seen how.

More importantly in the present context: a natural thought is that if, at a possible world, something is possible in any sense, it is logically possible. For some notions of possibility this seems right. We would expect any physical possibility to be a logical possibility. And if $X=P$ then everything is logically possible. ${ }^{15}$ Indeed, for any $\kappa \in K$, if $w R_{\kappa} w^{\prime}$ then $w R_{\lambda} w^{\prime}$.

But at least if $P$ is a proper subset of $W$, there are good reasons why this should not hold for all $\kappa \in K$. Consider epistemic possibility. Take a logical untruth, $A$, of enormous complexity: one which it would take longer than the history of the cosmos to decide. As far as is known, $A$ could be true. So there must be some $w \in X-P$, such that $@ R_{\varepsilon} w$.

Or again, suppose, for the sake of illustration, that the Law of Excluded Middle is a logical truth. Let us suppose that intuitionist critiques have been so fierce that we are now no longer sure whether $A$ is true or not, where, this time, $A$ is: either there are or there are not 17 consecutive 0 s in the decimal expansion of $\pi$. It could be false for all we know; but $A$ is false at no logically possible world, so there must be a $w \in X-P$, such that $@ R_{\varepsilon} w$. Or a more realistic example. Let $G$ be a statement of Goldbach's Conjecture. (Every even number greater than 2 is the sum of two primes.) The conjecture is currently undecided. It is true for all we know; it is false for all we know. Hence there are worlds $w_{1}$ and $w_{2}$, such that $G$ is true at $w_{1}$, false at $w_{2}$, and $@ R_{\varepsilon} w_{1}$ and $@ R_{\varepsilon} w_{2}$. Either $w_{1} \in X-P$ or $w_{2} \in X-P$.

Or consider deontic modality. I may promise to do something logically impossible (such as prove some mathematical statement which is, as a matter of fact, false). Or I may make promises to do incompatible things, such as to be in two different places at the same time (assuming such to be impossible). I am then morally obliged to do impossible things; ${ }^{16}$ that is, the worlds that realize my obligations are impossible. Hence, for any $w$ such that $@ R_{\delta} w$,

\footnotetext{
${ }^{15}$ As argued by Mortensen (1989). Even if $X$ is not $P$, this may still be the case. In $F D E$ and $L P$ everything is logically possible, because of the trivial possible world. The thought that every situation is logically possible may initially seem an odd one. But it should be remembered that logical possibility is a very weak constraint. Even if one is of a classical persuasion, it is a logical possibility that I can jump a kilometre into the air, that the moon is made of blue cheese, etc. Usually, when we are concerned with possibility, we are concerned with much more restricted notions, especially physical possibility.

${ }^{16}$ See Priest (1987), ch. 13.
} 
$w \in X-P$.

Sometimes, at this point, people will say things like 'Of course we are assuming an ideal agent'. A poor move. It helps us not one iota to understand what it means for us to know or be obliged to do something, if all we understand is what it is for God to know or be obliged to do it. We are interested in notions that apply to us: not God.

\subsection{The Secondary Directive}

The Primary Directive tells us that everything must hold at some world, and everything must fail as some world. There is, however, a stronger condition:

- If $A$ and $B$ are distinct formulas, there are worlds where $A$ holds and $B$ fails.

Let us call this the Secondary Directive. (It of course entails the Primary Directive.) The above semantics does not deliver this directive. Thus, for example, if $C$ is true at any $w \in X$, so is $C \vee D$, for any $C$ and $D$. More generally, if $A$ entails $B$ in FDE, and $A$ is true at $w$, so is $B$. Clearly, for some $A$ s and $B \mathrm{~s}$, a world that realises the Secondary Directive must be logically impossible. If one takes as a motto the thought that at an impossible world, anything can happen, the Secondary Directive seems entirely reasonable. Are there stronger reasons?

There are. One concerns conditionals with logically false antecendents. Thus, assuming that intuitionist logic is not the correct logic, the following seem true and false, respectively:

- If intuitionist logic is correct, the Law of Excluded Middle fails.

- If intuitionist logic is correct, the Law of Non-Contradiction fails.

Given something like a standard theory of counterfactuals,${ }^{17}$ to evaluate such conditionals we must consider worlds where intuitionist logic is correct. If $A$ does not entail $B$ in intuitionist logic, there must be such worlds where $A$ holds and $B$ fails. This does not happen in the present semantics. In intuitionist logic $\neg \neg A$ does not entail $A$; but in the present semantics the one holds at a world iff the other does. More generally, consider an arbitrary logic, $L$, and some $A$ which does not entail some $B$, according to $L$. Then to

\footnotetext{
${ }^{17}$ See Priest (2008), ch. 5.
} 
evaluate counterfactuals of the form: 'if $L$ were the correct logic...', we have to consider worlds where $L$ is the correct logic, and so where $A$ may hold and $B$ may fail. ${ }^{18}$

A second reason comes from consideration of intentional states, such as fear, hope, etc - and crucially in the current context, belief, knowledge, and conception. People being what they are, if $A$ and $B$ are distinct, someone may believe $A$, but not $B$. No one said that we are dealing only with rational agents - whatever that may mean. And, even a rational agent (not an idealised one) may believe/know all the axioms of Peano Arithmetic, without believing/knowing all their consequences. Hence we are required to countenance worlds which realise these states. The Secondary Directive delivers these.

How, technically, are we to obtain states delivered by the Secondary Directive? The simplest way is by brute force. We now augment $\mathrm{X}$ with a set of worlds, $Z$, to give us a set of worlds $W=X \cup Z$. At worlds in $Z$, every formula is treated as atomic. (Priest (2005) calls such worlds open worlds.) Thus, in an interpretation, $\nu^{+} / \nu^{-}$applies to arbitrary formulas (not just parameters). The truth conditions at worlds in $X$ are now as before (so if $A$ is not atomic, $\nu^{ \pm}(A)$ is irrelevant). But if $w \in Z$ :

- $w \Vdash^{+} A$ iff $w \in \nu^{+}(A)$

- $w \Vdash^{-} A$ iff $w \in \nu^{-}(A)$

And for $\kappa \in K, R_{\kappa}$ is a binary relation on $W$. The possible worlds are still $P \subseteq X$, and the impossible worlds are $(X-P) \cup Z$. @ is still in $P$. It is easy to see that the Secondary Directive (and so the Primary Directive) is now satisfied. ${ }^{19}$

At this point, one might think one has gone too far. Why does one need to countenance all these worlds? Here is a natural view. ${ }^{20}$ Counterfactuals are context dependent. If we consider counterfactuals such as 'If intuitionist logic were correct ...', the context requires us to consider worlds in which intuitionist logic is true; so we need to extend the realm of possibility to those worlds that are intuitionistically possible. Other contexts will require

\footnotetext{
${ }^{18}$ One notable exception: logics where the inference $A \vdash A$ fails.

${ }^{19} \mathrm{In}$ truth, it is only $\nu^{+}$that is required to deliver the Secondary Directive. We cannot give up $\nu^{-}$, though, since it may be involved in the falsity conditions of modal formulas at @; though this leaves us free to impose constraints on $\nu^{-}$if required for any reason.

${ }^{20}$ Suggested to me by Hartry Field.
} 
similar extensions. But there is no context in which we need to consider all such worlds. Conditionals are, of course, only one example of topics for which we need impossible worlds. But even concentrating just on conditionals, and granting the analysis given - at least for the sake of argument-even if it is the case that there is no context which requires every world, every world in $Z$ will be required in some context (since we may consider an arbitrary logic). Hence, globally, they must all be available to us. Whether one wants to call the worlds where intuitionist logic holds 'possible' in an extended sense, appears to me to me a terminological matter. Call them so if one wishes; but if intuitionist logic is not the correct logic, they may not be logically possible in the veridical sense.

\subsection{Generalising}

By taking advantage of the fact that the set of truths/falsehoods in any world in $X$ can be imitated by a world in $Z$, we can, in fact, make matters more uniform (and cut out the first stage in the construction of impossible worlds). An interpretation is now a tuple $\left\langle W, @,\left\{R_{\kappa}: \kappa \in K\right\}, \nu\right\rangle . W$ is a set of worlds; @ $\in W$; for $\kappa \in K, R_{\kappa}$ is a binary relation on $W ;^{21}$ and for any formula, $A, \nu^{ \pm}(A)$ are subsets of $W$. The truth conditions for @ are as in $2.1 / 2.2$, and the truth conditions for any other world, $w$, are as in 3.3. What are the possible worlds? We may simply take these to be those closed under the $S 5$ version of whichever of our four logics we hold to be correct (or if this is explosive, all such worlds except the trivial world). ${ }^{22}$

The techniques employed here are also generalisable in natural ways to most other standard propositional logics - not just the four we have met: $F D E, L P, K_{3}$, and classical logic. For example, if we take as our basic propositional logic an LFI, ${ }^{23}$ possible worlds have this as their underlying logic, and the negations of modal statements are given non-deterministic truth conditions. Since LFIs are paraconsistent logics, all worlds obtained in this way are possible. Or we may take a propositional logic that itself has world semantics. Thus, the Kripke semantics for intuitionistic logic adds a

\footnotetext{
${ }^{21}$ In fact, we do not need to consider what is accessed by non-@ worlds under $R_{\kappa}$, since the truth/falsity of modal sentences at such worlds is taken care of by $\nu$. We may therefore take $R_{\kappa}$ simply to be of the form $\{\langle @, y\rangle: y \in Y\}$ for some $Y \subseteq W$.

${ }^{22}$ And the new context may suggest some new members of $\bar{K}$, such as 'It is intuitionistically possible that ...'?

${ }^{23}$ See Carnielli, Coniglio, and Marcos (2007), and Bueno-Soler (2012).
} 
binary accessibility relation to give the truth conditions of the conditional. ${ }^{24}$ All the worlds delivered are possible. Or the Routley/Meyer world semantics of relevant logics add a ternary accessibility relation to give the truth conditions of the conditional. ${ }^{25}$ The semantics themselves specify possible (normal) and impossible (non-normal) worlds. In all cases, open worlds may be added to the models, in order to satisfy the Secondary Directive (and so the Primary Directive if it is not already satisfied.)

\section{Conceivability and Possibility}

I now want to turn to the notion of conceiving. ${ }^{26}$ Perhaps this can be understood in many ways. I intend to use conceive here as roughly synonymous with imagine: the sort of imagination employed by scientists, mathematicians, philosophers, novelists, political reformers, theologians, visionaries, and so on. ${ }^{27}$ In imagination, a state of affairs or an object is brought before the mind, and may be considered, enjoyed, its consequences thought through, and so on. 'Conceive' can be an intentional operator (to conceived that something), and it can be an intentional predicate (to conceive an object). Let us start with the intentional operator.

A very traditional view is that if one can conceive of something, it is possible. As David Hume put it: ${ }^{28}$

'Tis an establish'd maxim in metaphysics, That whatever the mind clearly conceives includes the idea of possible existence, or in other words, that nothing we imagine is absolutely impossible.

Hume's absolute impossibility here is essentially logical impossibility. ${ }^{29}$ Hence,

\footnotetext{
${ }^{24}$ See Priest (2008), ch. 6.

${ }^{25}$ See Priest (2008), ch. 10.

${ }^{26} \mathrm{OED}$, to conceive: 'to take or admit into the mind, to form in the mind, to grasp with the mind'.

${ }^{27}$ OED, to imagine: 'to form a mental image of, to represent to oneself in imagination, to create as a mental conception, to conceive'. There is one sense of the word according to which what is imagined 'should not be known with certainty' (OED, again). This is not the sense at issue here.

${ }^{28}$ Selby-Bigge and Nidditch (1978), p. 32.

${ }^{29}$ For Hume, for something to be absolutley impossible is for it to imply a contradiction. (See Lightner (1997), p. 115.) I take it that he holds that the negation of any "relation of ideas" would do this.
} 
for Hume, one cannot conceive of a logical impossibility. In particular, there are things that cannot be conceived.

Even given a Humean conception of what is logically impossible, I have always found this view incredible. Take Goldbach's conjecture again. I have no difficulty in conceiving this, and no trouble conceiving its negation, though one of these is mathematically impossible. Indeed, mathematicians must be able to conceive these things, so that they understand what it is of which they are looking for a proof, or so that they can infer things from them, in an attempted reductio proof. Nor does the conceivability of Goldbach's conjecture and its negation disappear if I discover which one of them is true, and so the other no longer appears mathematically possible to me. Hence, when something is conceived it may not even be appear to be possible. ${ }^{30}$

Similarly, the claim that intuitionist logic is true is, I take it, logically false (and if you disagree, merely replace this with classical logic in the following example). Yet I have no problem in conceiving what it would be like for it to be true. Indeed, I have to do so in order to be able to debate with intuitionists on the matter.

Moreover, I have no problem in imagining that deep in a trench at the bottom of the Pacific Ocean, there is a pearl which is round and square. I cannot form a visual image of this. But imagination should not be confused with visual imagery. I cannot form a visual image of a chiliagon (a regular 1,000 sided figure), even though there is nothing impossible about this. Conversely, I can visually picture a state of stationary motion, even though this is contradictory. This occurs in the well-know "waterfall illusion". In this, one conditions the visual system with constant motion in one direction. The after-image will make things appear to be moving in the opposite direction. But if one focusses on a point in the visual field, it appears to be stationary, even though in motion. ${ }^{31}$

And again, understanding a work of fiction requires an act of imagination. Yet there are works of fiction with essentially inconsistent plots - for example,

\footnotetext{
${ }^{30}$ See Yablo (1993). Yablo's own account of conceivability (in Section 10) is that $A$ is conceivable if one can imagine a world that verifies $A$. In fact, I agree with this, since I take everything to be conceivable/imaginable. This is not what Yablo intends, however. For, by 'world', he means '(classically) possible world'. Yablo tells us (p. 30) that one cannot imagine, e.g., tigers that lick all and only those tigers that do not lick themselves. I find this no harder to imagine than a set that contains all those sets which are not members of themselves. (And I could imagine this even before I became a dialetheist.)

${ }^{31}$ For discussion and references, see Priest (2006). 3.3.
} 
Sylvan's Box. ${ }^{32}$ One must therefore be able to imagine such things.

Indeed, it seems to me that I can conceive of and imagine anything that can be described in terms that I understand ${ }^{33}$ (Which is not to say that such things are the only things I can imagine. That is another matter.) In fact, such understanding allows for the possibility of conception - which is not the same as the conception of possibility. To conceive, I merely have to bring the state of affairs, so described, before the mind.

So, to return to the formal semantics, given any of the interpretations with impossible worlds of the kinds described in 3.3 , there will be a really most general notion of possibility: being true at some world. Call this global possibility, $\gamma$. If $W$ is the set of all worlds, then for @ (or, more generally, for every world in $P$ ), @ $R_{\gamma} w$ for all $w \in W$. Given the Primary Directive, for any $A,\langle\gamma\rangle A$ is true at @, and $[\gamma] A$ is not true at @. We may take $\gamma$ to be the modality of conception/imagination: $\langle\gamma\rangle A$ is ' $A$ is conceiveable/imaginable'.$^{34}$ I should note that, strictly speaking, conceivability is agent-relative (as is knowability). In particular, the $A$ s in question have to come from a language that the agent in question understands (in a way that, say, a medieval monk could not understand the language of quantum mechanics). ${ }^{35}$

Three objections. One. It might be suggested that if I seem to conceive of

\footnotetext{
${ }^{32}$ Priest (1997b).

${ }^{33}$ There is a somewhat thorny issue here about what it is, exactly, to understand. Can a congenitally blind person understand the predicate 'is red', for example? I am inclined to the view that they can, if they can use the word - by whatever means - in a roughly normal way. When they imagine something red, the phenomenological content may, however, be quite different from that of a sighted person who imagines something red.

${ }^{34}$ Semantics for a logic of imagination can be found in Niiniluoto (1987), Costa-Leite (2010), and Wansing $(201+)$. These are all variations on possible-world semantics, and hence do not allow for imagining the impossible. Even worse, they all require imagination to satisfy certain logical closure conditions. Thus, they all validate the principle that if $A$ is imagined, and $A$ is logically equivalent to $B$, then $B$ is imagined. This is clearly incorrect. $A$ is logically equivalent to $(A \wedge C) \vee A$, but I can imagine that Sherlock Holmes lived in Baker St without imagining that (Sherlock Holmes lived in Baker St and $E=m c^{2}$, or Holmes lived in Baker St). Nothing about Special Relativity need have crossed my mind at all. It is precisely this to which the Secondary Directive caters. Berto (2012) ch. 7 has a semantics for conception/representation which uses impossible worlds. He does not requre that everything be conceivable, but the semanatics does allow for that possibility.

${ }^{35}$ One might also doubt that a person understands indefinitely long sentences of such a language. By the same token, one might doubt that such sentences are really grammatical. One might therefore be inclinded to put the same bounds of finitude on both both.
} 
(imagine) something that is impossible, I am, in fact, conceiving something else. Thus (assuming that identities are necessary), when I conceive that water is not $\mathrm{H}_{2} \mathrm{O}$, what $\mathrm{I}$ am actually conceiving is that some substance that is a colourless, odourless, potable liquid - even called 'water' - is not $\mathrm{H}_{2} \mathrm{O}$. ${ }^{36}$ Of course, I can imagine that too; but that is not what I am imagining when I imagine that water is not $\mathrm{H}_{2} \mathrm{O}$ : I am imagining something about water. The imagination is de re. In the same way, when I imagine that Sarah Palin was the US Vice President after the 2012 US election, I am imagining something about Palin. When I imagine that Routley found a box that was empty and not empty, it is him that I imagine. And when I imagine that 361 is a prime number (it isn't) I am imagining something about that very number.

Two. It might be suggested that this is not the notion of conceivability operative in Hume's dictum, since one who imagines impossibilities is not clearly conceiving. If one takes it that one can clearly conceive only what is logically possible, this turns Hume's dictum into an empty tautology - and a useless one, since we may not know what is impossible in this sense. If one is using the word in a more common-sense way, it is something of an insult to say that a logician or mathematician who conceives of impossibilities is not conceiving these things clearly, since it is tantamount to an accusation of confusion. Perhaps, there is some other notion of conceivability that satisfies Hume's dictum, and which can serve as a test for possibility. If so, I leave it to others to articulate it. I know of no satisfactory such articulation. ${ }^{37}$

Three. It might be suggested that I am confusing imagination with supposition. One can suppose anything; this does not mean that one can imagine anything. One can indeed suppose anything, but I am not talking about supposition. To suppose something is to assume it, usually for the purpose of drawing conclusions. ${ }^{38}$ Imagining does not require this. I ask you to imagine that George Bush likes dressing up in a tutu. I am not asking you to suppose anything, or infer anything - merely to use your imagination. Indeed, we will

\footnotetext{
${ }^{36}$ See Berto (2012), 6.3.2.1 for references and discussion.

${ }^{37}$ Chalmers (2002) constructs an eightfold taxonomy of notions of conceivability, and argues that at least one of these entails possibility: ideal primary positive conceivability. This may well be different from the notion of conceivability I am discussing here - though the circularity in his glosses of these notions make me less than certain. But in any case, one thing is clear: the ideality involved is that of some infinite and infallible a priori reasoner - not a very useful notion for mere mortals.

${ }^{38}$ OED, to suppose: 'to think or assume that something is true or probable but lack proof or certain knowledge', 'used to introduce a hypothesis and imagine its development'.
} 
next turn to imagining objects. These are not even the kind of thing that can be supposed.

So let us turn to the intentional predicate. For an agent to conceive of an object (de re) is simply for them to bring before the mind a term, $t$, which refers to it. ${ }^{39}$ And just as I can conceive of any state of affairs I can describe, I can conceive of any object I can describe, even if it is an impossible one. That is, anything of the form $\langle\varphi\rangle m C t$ is true (at the actual world) -where $m$ is me, $t$ is any name or description, and $x C y$ is ' $x$ conceives of $y$ '.

It is natural to ask, at this point, what the difference is between a possible object and an impossible one-or better which conditions characterise possible objects and which conditions characterise impossible ones.

Let $A$ be any condition with one free variable, $x$. Then a condition is possible if there is a possible world, $P$, where something satisfies $A$ in $P$. Otherwise it is impossible. (By the Primary Directive, any condition is satisfied at some worlds.) Thus, if one takes classical logic to be correct, and $A$ is an inconsistent condition, then it will be an impossible one, and $\varepsilon x A$ will be an impossible object. ${ }^{40}$

Finally, how are matters affected if the Primary Directive is satisfied in possible worlds (for example, if the correct logic is $F D E$ )? Then every state of affairs is logically possible. By the same token, every condition is realised in a possible world, so there are no impossible conditions. So whatever $A$ is, $\varepsilon x A$ is a possible object. If this is the case, there is a certain irony here. One must agree with the quote from Hume! If everything is logically possible, then anything 'the mind clearly conceives' is logically possible! Of course, that is not what he meant. Perhaps there is a lesson here. ${ }^{41}$

\section{Conclusion}

The Norweigan explorer Fridtjof Nansen said: 'The difficult is what takes a little time; the impossible is what takes a little longer. ${ }^{42}$ Philosophy plays the long game. The impossible has always been a marginalised character in Western philosophy. The infinite had always been a marginalised character in mathematics until the time of Cantor. But just as Cantor pro-

\footnotetext{
${ }^{39}$ See Priest (1995), 4.8. Again, I am assuming that the agent understands the term ' $t$ '.

${ }^{40}$ Here, $\varepsilon$ is the indefinite description operator: a (particular) object such that.

${ }^{41}$ As the old saying goes: be careful of what you wish for; you might just get it.

${ }^{42}$ Cohen and Cohen (1992), p. 291.
} 
vided an understanding of the mathematical structure of the infinite, modern logic - especially paraconsistent logic - has provided an understanding of the mathematical structure of the impossible. One can hardly pretend that this is an achievement on the scale of Cantor's (at least so far). However, I think that it has the potential to open people's eyes in the same way. Maybe even wider. $^{43}$

\section{References}

[1] Berto, F. (2012), Existence as a Real Property, Dordrecht: Springer, Synthese Library.

[2] Berto, F. (2013), 'Impossible Worlds', in E. Zalta (ed.), Stanford Encyclopedia of Philosophy, http://plato.stanford.edu/entries/impossibleworlds/.

[3] Bueno-Soler, J. (2012), 'Models for Anodic and Cathodic Multimodalities', Logic Journal of the IGPL 20: 458-476.

[4] Carnielli, W., Coniglio, M., and Marcos, J. (2007), 'Logics of Formal Inconsistency', pp. 1-93, Vol. 14, 2nd ed., of D. Gabbay and F. Guenthner (eds.), Handbook of Philosohical Logic, Dordrecht: Kluwer Academic Publishers.

[5] Carnielli, W., and Pizzi, C. (2008), Modalities and Multimodalities, Berlin: Springer.

[6] Chalmers, D. (2002), 'Does Conceivability Entail Possibility?', ch. 3 of T. Szabó and J. Hawthorne (eds.), Conceivability and Possibility, Oxford: Oxford University Press.

[7] Cohen, J. M. and M. J. (1992), The New Penguin Dictionary of Quotations, London: Penguin.

\footnotetext{
${ }^{43}$ Versions of this paper were given at the Fordham University Metaphysics and Mind Group, the Departments of Philosophy at the University of Amsterdam and the Australian National University, and the conference Thinking the Impossible, at the University of Turin. Thanks go to those present for their helpful comments. Thanks, too, go to Hartry Field for comments on an earlier draft.
} 
[8] Costa-Leite, A. (2010), 'Logical Properties of Imagination', Abstracta 6: $103-16$.

[9] Garson, J. (2014), 'Modal Logic', in E. Zalta (ed.), Stanford Encyclopedia of Philosophy, http://plato.stanford.edu/entries/logic-modal/.

[10] Knuuttila, S. (2013), 'Medieval Theories of Modality', in E. Zalta (ed.), Stanford Encyclopedia of Philosophy, http://plato.stanford.edu/entries/modality-medieval/.

[11] Lightner, D. T. (1997), 'Hume on Conceivability and Inconceivability', Hume Studies 23: 113-32.

[12] Menzel, P. (2013), 'Possible Worlds', in E. Zalta (ed.), Stanford Encyclopedia of Philosophy, http://plato.stanford.edu/entries/possible-worlds/.

[13] Mortensen, C. (1989), 'Anything is Possible', Erkenntnis 30: 319 - 337.

[14] Niiniluoto, I. (1985), 'Imagination and Fiction', Journal of Semantics 4: $209-22$.

[15] Priest, G. (1987), In Contradiction, Dordrecht: Martius Nijhoff; 2nd ed., Oxford: Oxford university Press, 2006.

[16] Priest, G. (1995), Beyond the Limits of Thought, Cambridge: Cambridge University Press; 2nd ed., Oxford: Oxford University Press, 2002.

[17] Priest, G. (ed.) (1997a), Notre Dame Journal of Formal Logic 38 (4). (A special issue on impossible worlds.)

[18] Priest, G. (1997b), 'Sylvan's Box', pp. 573-82 of Priest (1997a); reprinted as 6.6 of Priest (2005).

[19] Priest, G. (2005), Towards Non-Being: the Logic and Metaphysics of Intentionality, Oxford: Oxford University Press.

[20] Priest, G. (2006), Doubt Truth to be a Liar, Oxford: Oxford University Press.

[21] Priest, G. (2008), Introduction to Non-Classical Logic: From If to Is, 2nd ed., Cambridge: Cambridge University Press. 
[22] Selby-Bigge, L., and Nidditch, P. (eds.) (1978), A Treatise of Human Nature, 2nd ed., Oxford: Clarendon Press.

[23] Smith, R. (2011), 'Aristotle's Logic', in E. Zalta (ed.), Stanford Encyclopedia of Philosophy, http://plato.stanford.edu/entries/aristotle-logic/.

[24] Wansing, H. (201+), 'Remarks on the Logic of Imagination', Synthese, to appear.

[25] Yablo, S. (1993), 'Is Conceivability a Guide to Possibility?', Philosophy and Phenomenological Research, 53: 1-42. 


\section{University Library}

\section{- M M N E R VA A gateway to Melbourne's research publications}

Minerva Access is the Institutional Repository of The University of Melbourne

Author/s:

Priest, G

Title:

Thinking the impossible

Date:

2016-10-01

Citation:

Priest, G. (2016). Thinking the impossible. PHILOSOPHICAL STUDIES, 173 (10), pp.2649-2662. https://doi.org/10.1007/s11098-016-0668-5.

Persistent Link:

http://hdl.handle.net/11343/282590 